\title{
LES PEUPLEMENTS DE POISSONS DE L'ANNÉE DE QUELQUES TYPES D'ANNEXES FLUVIALES DANS LA PLAINE DE LA BASSÉE (SEINE).
}

\author{
E. TALES, P. BOËT, R. BERREBI DIT THOMAS, \\ avec la collaboration technique de A. RUMEAU.
}

CEMAGREF, division qualité des eaux,

14 avenue de Saint-Mandé, 75012 Paris, France.

\section{RÉSUMÉ}

Dans un secteur de la Seine moyennement aménagé, les peuplements de poissons de l'année sont étudiés simultanément dans le chenal principal ainsi que dans cinq annexes fluviales de différents types. Six campagnes de pêche électrique réalisées durant l'été 1994 ont permis la capture de 3302 poissons de l'année représentant 18 espèces. L'analyse des abondances des espèces montre un gradient spatial, des milieux lotiques aux milieux lentiques. Elle met également en évidence l'évolution saisonnière subie par les peuplements de poissons de l'année. En analysant les préférences des espèces vis-à-vis de chaque milieu, au regard de leur appartenance aux groupes de reproduction, ce gradient spatial des annexes est affiné et, en particulier, singularise les annexes d'origine anthropique. Ainsi, cette étude montre la complémentarité entre, d'une part, les annexes et le chenal principal et, d'autre part, entre les différents types d'annexes vis-à-vis de la reproduction des espèces de poissons d'un cours d'eau de plaine alluviale.

Mots-clés : reproduction, habitat, plaine alluviale, aménagement.

\section{YOUNG-OF-THE-YEAR FISH ASSEMBLAGE IN SEVERAL BACKWATERS OF THE SEINE RIVER FLOODPLAIN.}

\begin{abstract}
In a middle reach of the Seine river, young-of-the-year fish assemblage is studied both in the main channel and in five backwaters from natural or artificial origin, characterized by geomorphologic type. A total of 3302 specimens representing 18 species was collected with electrofishing from late June to September 1994. Principal Components Analysis is performed on the table of species abundances per station. Opposing typical rheophilic species (as Barbus barbus, Chondrostoma nasus, Leuciscus leuciscus) to limnophilic or eurytopic ones (as Lepomis gibbosus, Rhodeus amarus, Alburnus alburnus, Rutilus rutilus), shows a spatial gradient, from lotic to lentic sites. Seasonal evolution of young-of-the-year fish assemblage is also demonstrated. Then, ecological profiles of species versus study sites are performed to precise this spatial gradient. Considering species ecological preferences and reproductive guilds, it is possible to discriminate artificial backwaters, characterized by a species (Lepomis gibbosus) pertaining to nest-guarders reproductive guild. Thus, this study shows the complementarity between backwaters and the main channel, and between the different backwaters types for fish species recruitment in a floodplain river.
\end{abstract}

Key-words : reproduction, habitat, floodplain, management. 


\section{INTRODUCTION}

Les liens existant entre un fleuve et sa plaine alluviale génèrent une variété de milieux annexes qui constituent sa dimension latérale (RICHARDOT-COULET et al., 1982). Leurs rôles particuliers vis-à-vis des peuplements piscicoles ont déjà été mis en évidence. Ils leur servent de refuge thermique (BOUVET et al., 1985) et d'abris en cas de crue (HOLCIK, 1988). Ils constituent des lieux de nutrition (REIMER, 1991) ou des sources d'aliments qui enrichissent le chenal principal (SCHIEMER et al., 1991). Enfin, ils se sont révélés être des lieux propices à la reproduction des poissons (ANTIPA, 1911; COPP et PENAZ, 1988; SCOTT et NIELSEN, 1989) et des sites de nursery pour les alevins (CARREL, 1986 ; SHEAFFER et NICKUM, 1986).

L'anthropisation croissante des hydrosystèmes, qui débute souvent par la régulation et la chenalisation du fleuve, tend à réduire voire supprimer les connexions latérales du chenal avec ses milieux annexes et annihile leur rôle (HOLCIK, 1990 ; SCHIEMER et WAIDBACHER, 1992 ; PATTON et HUBERT, 1993 ; SCHEIDEGGER et BAIN, 1995). Néanmoins, quelques études semblent indiquer que de nouveaux milieux artificiels générés par les aménagements peuvent se substituer aux annexes naturelles pour remplir un rôle comparable vis-à-vis de la reproduction des espèces piscicoles (SABO et KELSO, 1991 ; POIZAT, 1993). Mais la plupart de ces travaux sont menés dans des contextes opposés, où ne coexistent pas à la fois les milieux annexes naturels et artificiels. II est alors difficile d'estimer quel type de milieu conditionne la reproduction de certaines espèces de poissons, phase-clé de leur cycle vital.

Les peuplements de poissons de l'année et leur structure sont un indice de l'efficacité de la reproduction (SCHEFFEL et SCHIRMER, 1991). Ils s'avèrent être des descripteurs pertinents et fonctionnels de l'ichtyofaune (COPP et al., 1991). En effet, les jeunes alevins demeurent sur le lieu de leur naissance avant de se disperser vers l'ensemble du milieu au bout de quelques mois, au stade juvénile. Ainsi, en raison de ce rôle indicateur, l'étude de leurs peuplements, menée durant la saison estivale, permet de comparer les potentialités de différents types d'annexes vis-à-vis de la reproduction des espèces piscicoles. Dans cet objectif, la présente étude est menée sur les peuplements de poissons de l'année de plusieurs types d'annexes fluviales.

\section{MATÉRIEL ET MÉTHODES}

\section{Site d'étude}

Le secteur étudié est situé dans le cours moyen de la Seine, à l'aval de la confluence de l'Aube, où débute la région de la Bassée constituée par une large plaine alluviale (fig. 1). La Seine, en ordre 5, fait en moyenne une centaine de mètres de large et 4 mètres de profondeur. La qualité physico-chimique générale de l'eau, appréciée à partir de cinq paramètres (oxygène dissous, Demande Biologique en Oxygène, Demande Chimique en Oxygène, ammoniac et Matières en Suspension), est bonne (classe 1B, DIREN CHAMPAGNE-ARDENNE, 1995), déclassée ponctuellement par des teneurs en Matières en Suspension élevées. Ce secteur a été aménagé anciennement pour la navigation, mais n'est plus emprunté depuis la fermeture du canal de la Haute-Seine en 1957, alors interdit à tout trafic. Il est généralement considéré comme sub-naturel, car il demeure caractéristique de l'évolution morphologique de la Seine dans sa plaine alluviale. Son aménagement s'est essentiellement traduit par un recoupement des méandres les plus accentués et le creusement de la partie centrale du chenal. Par ailleurs, aucune intervention n'a été menée sur les berges si ce n'est quelques enrochements très localisés. Néanmoins, un barrage subsiste et délimite l'aval du secteur. De nombreux milieux annexes, en communication permanente avec le chenal principal, sont recensés dans ce secteur et témoignent non seulement de l'évolution morphologique naturelle du fleuve mais aussi des actions anthropiques plus récentes. 


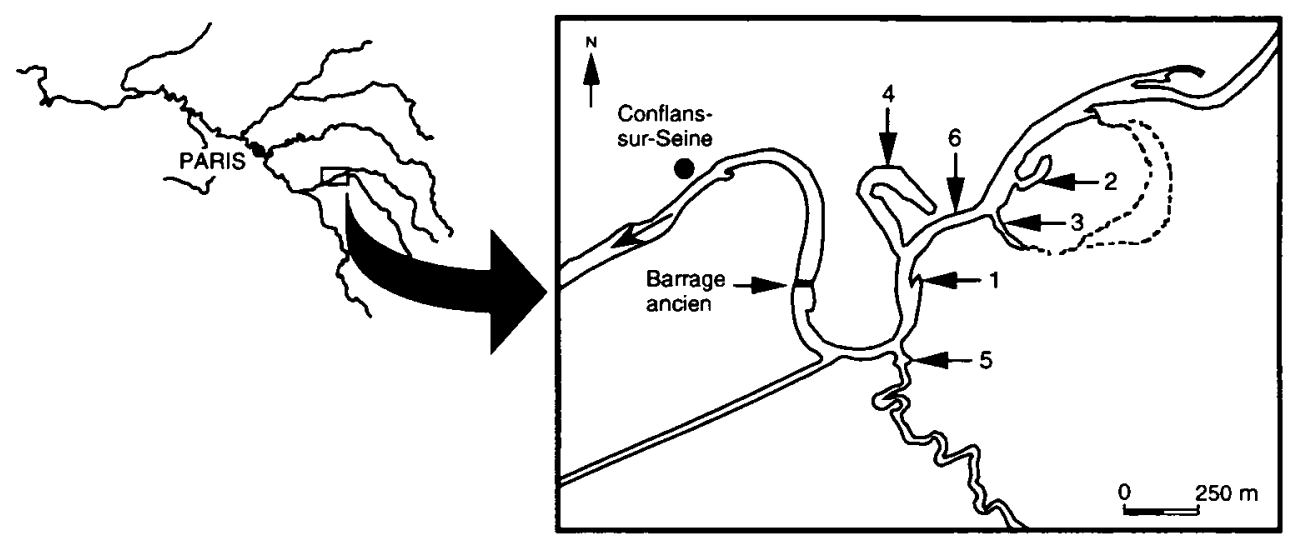

Figure 1 : Situation des stations d'étude.

Figure 1 : Location of the study sites.

Cinq milieux annexes ont été retenus. Ils ont été choisis pour leur représentativité, sur la base d'une typologie géomorphologique des chenaux secondaires d'écoulement de la plaine alluviale (DZANA, 1992). Ils présentent des caractéristiques morphométriques différentes (Tab. I). En revanche, leurs caractéristiques physiques sont peu contrastées et les habitats sont relativement homogènes parmi les stations. Les abris ligneux, la végétation aquatique ainsi que la ripisylve sont présents dans chacun des sites. Les substrats, de granulométrie plutôt fine, sont caractéristiques des zones de dépôt. Ces milieux sont d'origine naturelle $(1,2$ et 3$)$ ou anthropique (4 et 5). Les trois premiers milieux sont liés à des déplacements naturels, de plus ou moins grande ampleur, du lit mineur de la Seine : les stations 1 et 2, de faible taille, sont des dépressions de convexité, alors que la station 3 est un méandre délaissé dont la partie amont est comblée. La station 4 est un ancien méandre recoupé artificiellement, ce qui lui confère des caractéristiques morphométriques proches de celles du chenal. La station 5 est l'exutoire élargi d'un chenal secondaire, aménagé en bras de dérivation de moulin à partir du Moyen-Age. Seule cette dernière station est ouverte à l'amont. Enfin, le secteur du chenal principal considéré constitue la station 6 .

Tableau 1 : Caractéristiques morphométriques et biologiques des stations d'étude.

Table I : Morphometric and biological description of study sites.

\begin{tabular}{|c|c|c|c|c|c|c|c|}
\hline & Stations & 1 & 2 & 3 & 4 & 5 & $\begin{array}{c}6 \\
\text { (chenal) }\end{array}$ \\
\hline \multirow[t]{4}{*}{$\begin{array}{l}\text { Caractéristiques } \\
\text { morphométriques }\end{array}$} & $\begin{array}{l}\text { Communication } \\
\text { amont }\end{array}$ & non & non & non & non & oui & - \\
\hline & $\begin{array}{l}\text { Largeur de la } \\
\text { communication } \\
\text { aval (en mètres) }\end{array}$ & 20 & 5 & 5 & 45 & 10 & - \\
\hline & Superficie $\left(\mathrm{m}^{2}\right)$ & 2100 & 3150 & 2100 & 18300 & 2100 & - \\
\hline & $\begin{array}{l}\text { Profondeur } \\
\text { maximale }(\mathrm{m})\end{array}$ & 0,5 & 0,6 & 0,7 & 2,5 & 1,5 & 4 \\
\hline $\begin{array}{l}\text { Caractéristiques } \\
\text { vis-à-vis du } \\
\text { courant }\end{array}$ & & lentique & lentique & lentique & lentique & lotique & lotique \\
\hline \multirow[t]{2}{*}{$\begin{array}{l}\text { Caractéristiques } \\
\text { du peuplement }\end{array}$} & $\begin{array}{l}\text { Richesse } \\
\text { spécifique }\end{array}$ & 13 & 13 & 14 & 15 & 9 & 14 \\
\hline & $\begin{array}{l}\text { Diversité } \\
\text { (Shannon) }\end{array}$ & 2,77 & 2,47 & 2,94 & 2,76 & 2,51 & 2,9 \\
\hline
\end{tabular}




\section{Echantillonnage}

Le peuplement piscicole des six stations a été échantillonné lors de six campagnes, du 27 juin au 19 septembre 1994, afin de tenir compte de l'étalement de la période de reproduction de la plupart des espèces présentes sur le secteur, c'est-à-dire d'avril jusqu'en juillet, selon les conditions thermiques de l'année.

Les poissons ont été capturés par pêche électrique, selon la méthode des EPA (Echantillonnage Ponctuel d'Abondance, NELVA et al., 1979). Cette méthode consiste à effectuer des points de pêche, choisis de manière aléatoire et en nombre suffisant pour constituer un échantillon représentatif de la station. Le nombre de points de pêche par station (de 6 à 20) est proportionnel à sa superficie, de façon à ce que l'effort de pêche soit équivalent pour chaque station.

L'échantillonnage a été effectué à l'aide d'un appareil portable "Martin-Pêcheur ", qui délivre un courant impulsionnel de fréquence fixe. La tension de sortie et la fréquence ont été réglées de manière optimale $(200 \mathrm{~V}, 400 \mathrm{~Hz})$, compte tenu de la gamme de conductivité des eaux des milieux étudiés (de 350 à $450 \mu \mathrm{S}$ ). Ce matériel a été adapté pour la capture de poissons de petite taille en utilisant une anode circulaire de faible diamètre $(10 \mathrm{~cm}$, selon COPP, 1989a). Ainsi, son efficacité a été accrue pour des poissons dont la longueur standard est inférieure à $50 \mathrm{~mm}$, ce qui correspond globalement aux stades précoces de toutes les espèces.

En raison de la faible profondeur de la plupart des milieux étudiés, l'approche des points de pêche a été effectuée en bateau pneumatique de manière discrète pour limiter la fuite des poissons. A chaque point, après immersion de l'anode pendant 30 secondes, les poissons ont été récupérés à l'aide d'une épuisette munie d'un filet de maille de $1 \mathrm{~mm}$ en veillant à recueillir également les individus susceptibles d'être captifs dans les touffes de végétaux.

Les alevins ainsi capturés ont été fixés dans du formol à $5 \%$, pour être déterminés au laboratoire sous une loupe binoculaire. Les stades observés s'échelonnent de la larve (ptérygiolarve) aux premiers stades juvéniles (sensu BALON, 1975b). Leur identification, parfois délicate, a été facilitée en procédant de manière rétroactive, c'est-à-dire en commençant par les individus les plus âgés, capturés lors de la dernière campagne, en utilisant des clés de détermination spécifiques (BALINSKY, 1948 ; KOBLITSKAIA, 1981 ; SPINDLER, 1988 ; MOOIJ, 1989). Les alevins identifiables sur place ou les adultes attirés en raison de leur proximité de l'anode ont été immédiatement mesurés et remis à l'eau.

La méthode utilisée paraît la mieux adaptée. Outre sa facilité de mise en oeuvre sur le terrain, car elle nécessite à la fois peu de personnel et peu de temps, elle présente une efficacité équivalente quel que soit le stade de développement des jeunes poissons, des larves aux juvéniles, contrairement à d'autres échantillonnages tels filets ou pièges, la capturabilité des poissons diminuant alors avec leur taille. Par ailleurs, cette méthode permet l'obtention de nombreux échantillons de petite taille, ce qui renforce la validité statistique des résultats (CYR et al., 1992) et élimine les problèmes d'épuisement des populations des milieux de taille réduite, inhérents à l'intensité du suivi saisonnier. C'est, enfin, une méthode standardisée qui fournit des données quantitatives fiables.

Néanmoins, la prospection par pêche électrique s'avère inefficace dans des profondeurs d'eau excédant $1 \mathrm{~m}$ environ et parfois est ainsi limitée à la zone rivulaire.

\section{Analyses statistiques}

Toutes les analyses ne concernent que les poissons de l'année. Leurs abondances ont été traitées par analyse multivariée à l'aide de la programmathèque ADE (CHESSEL et DOLÉDEC, 1991). L'Analyse en Composantes Principales (ACP) a été choisie, car elle s'avère particulièrement adaptée pour traiter des données quantitatives. Les effectifs des poissons de l'année ont été soumis, au préalable, à quelques transformations. L'objectif étant d'appréhender les résultats à l'échelle du secteur, les effectifs par point de pêche ont été regroupés par stationdate, ce qui représente 36 relevés ( 6 stations $\times 6$ dates). Afin d'éliminer l'effet attribuable 
à la taille des stations en fonction de laquelle varie le nombre de points de pêche réalisés, le contenu de chaque relevé a été ramené à 15 points de pêche. Ces effectifs ont enfin subi une transformation logarithmique $\left(\log _{2}\right)$ pour atténuer la prépondérance de certains échantillons.

Afin de préciser l'importance des six stations par rapport à chaque espèce, les profils écologiques spécifiques ont été calculés et testés. lls permettent de définir les préférences d'une espèce vis-à-vis d'un facteur déterminé (ici, les stations), par calcul de l'écart entre la fréquence de cette espèce dans les relevés d'une station et la fréquence de cette espèce dans l'ensemble des relevés (DAGET et GODRON, 1982). Les fréquences des espèces ont été calculées à partir des effectifs dans les points de pêche, afin d'en améliorer leur estimation. La valeur positive du profil d'une espèce indique sa sur-représentation tandis qu'une valeur négative traduit sa sous-représentation. La signification statistique de cette valeur est testée au moyen d'un Khi 2.

\section{RÉSULTATS}

Au total, 466 points de pêche ont été effectués dont 289 contiennent au moins un poisson de l'année. Le total des captures comprend 3302 individus représentant 18 espèces et appartenant à 6 familles (Tab. II). Globalement, la composition spécifique du peuplement de l'année, à dominante cyprinicole, est conforme au peuplement adulte du secteur. L'espèce la plus abondante est le chevaine, et les cyprinidés rhéophiles sont bien représentés. La présence du vairon est surprenante dans ce secteur de la Seine, qui doit constituer la limite aval de sa répartition. II faut souligner également l'importance de la bouvière, au troisième rang d'abondance, alors que cette espèce est d'habitude plutôt rare dans les inventaires de peuplements piscicoles adultes. II est vrai que la méthode d'échantillonnage employée est particulièrement adaptée à la capture des poissons de petite taille. En revanche, certaines espèces sont faiblement représentées dans ce peuplement de jeunes de l'année, comme le brochet. Sa reproduction étant précoce, les jeunes brochetons sont déjà de grande taille quand débute la période d'échantillonnage, ce qui explique leur faible abondance dans les captures. Le poisson-chat est également peu présent, en raison du comportement très grégaire des alevins dont la formation en boule leur permet vraisemblablement d'être évités par la méthode de pêche par point.

Tableau II : Liste des espèces de poissons de l'année capturées lors des six campagnes. Effectifs bruts totaux et par station. Appartenance des espèces aux groupes de reproduction (d'après Balon, 1975a) et aux groupes écologiques (d'après SCHIEMER et WAIDBACHER, 1992).

Table II : List and abundances of young-of-the-year fish species captured at all the dates. Reproductive guilds (after BALON, 1975a) and ecological guilds (after SCHIEMER and WAIDBACHER, 1992) of young-of-the-year fish species.

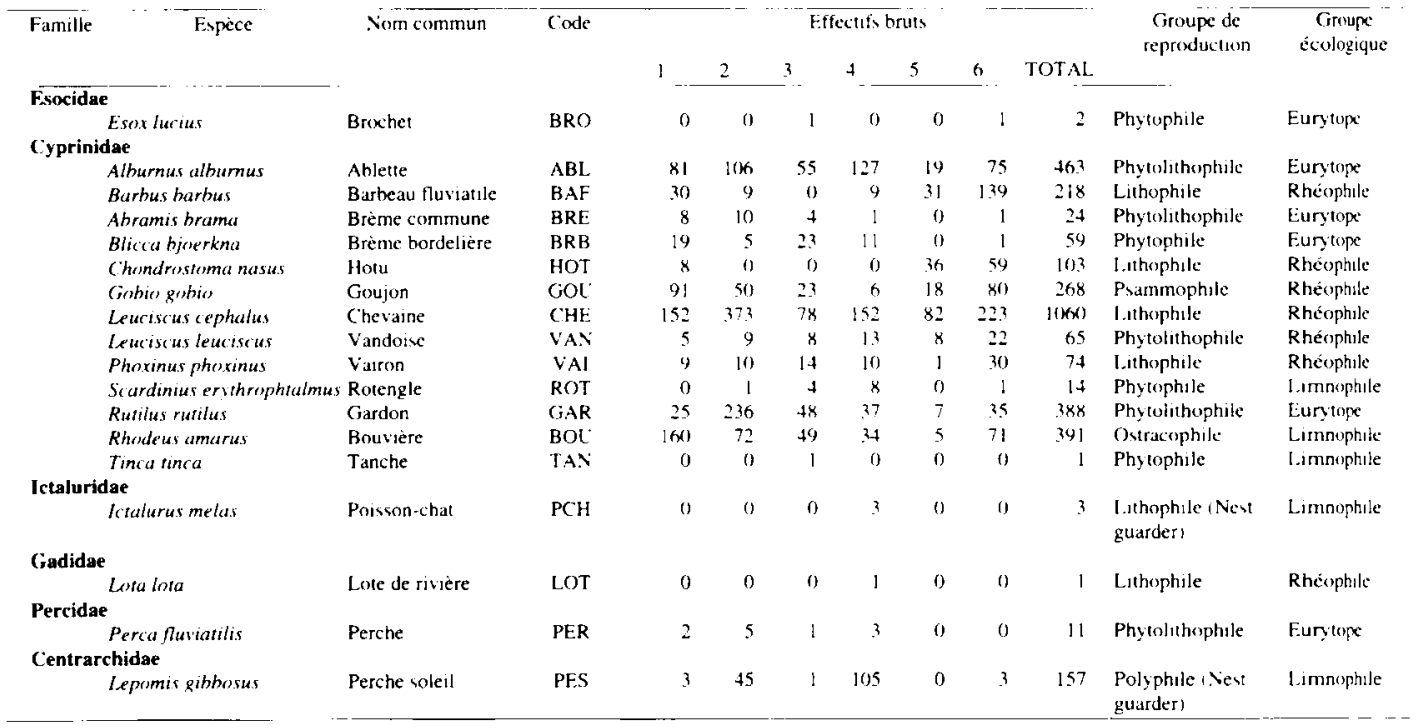




\section{Richesse spécifique et diversité des milieux}

La richesse spécifique des stations étudiées oscille entre 9 et 15 espèces (Tab. I) et est globalement corrélée avec la taille des milieux étudiés. Néanmoins, elle est comprise entre 13 et 15 espèces pour cinq des six stations. La station 5 , qui comporte 9 espèces, est de taille réduite mais présente un écart important avec la station 1, qui est de taille comparable et compte 13 espèces. Toutefois, la station 5 est la seule annexe lotique parmi toutes celles étudiées, ce qui constitue peut-être un facteur limitant vis-à-vis de certaines espèces. La station 4 , dans laquelle 15 espèces ont été capturées, est la plus grande. Le chenal principal a globalement une richesse spécifique comparable à ses annexes hydrauliques.

La diversité des milieux, déterminée à partir de l'indice de Shannon, est en revanche indépendante de leur taille. Le maximum est obtenu à la station 3 et le minimum à la station 5 . La diversité du chenal est intermédiaire.

\section{Abondance des poissons de l'année}

Les espèces représentées par moins de 5 individus (Esox lucius, Tinca tinca, Ictalurus melas, Lota lota) ont été éliminées du jeu de données. Le tableau faunistique soumis à une ACP, qui rassemble les abondances des espèces dans les relevés, se compose alors de 14 colonnes et 36 lignes (espèces $x$ relevés).

Le graphe des valeurs propres (fig. $2 \mathrm{~A}$ ) indique une structuration des données essentiellement sur les trois premiers axes qui représentent $63,4 \%$ de la variance totale du tableau. Les représentations successives des espèces et des relevés dans les différents plans factoriels permettent d'interpréter les trois axes retenus. En particulier, la représentation des relevés sous forme de graphique en étoile (fig. 2C et 2D) matérialisant les centres de classe, s'avère intéressante pour l'évaluation du positionnement de chaque classe (station ou date) dans les différents plans factoriels.

Le premier axe $\mathrm{F} 1$, qui extrait $31,3 \%$ de la variance totale du tableau faunistique, révèle un effet taille inhérent à la méthode d'analyse employée. Le long de cet axe s'ordonnent les abondances de toutes les espèces qui lui sont corrélées positivement (fig. 2B). Seule la perchesoleil y contribue négativement. En effet, l'abondance de cette espèce évolue à l'inverse de celle des autres et croît de la troisième campagne à la dernière tandis que, dans le même temps, les abondances de toutes les autres espèces ont tendance à régresser. Ce phénomène est imputable à la reproduction tardive de la perche-soleil relativement aux autres espèces.

Le deuxième axe F2 (18,6\% de la variance totale du tableau), indépendant du premier, permet d'ordonner les espèces selon le degré de rhéophilie défini pour les adultes (groupes écologiques sensu SCHIEMER et WAIDBACHER, 1992). II oppose les espèces typiquement rhéophiles (le barbeau, le hotu et la vandoise), aux espèces lénitophiles (la perche-soleil, la bouvière) ou eurytopes (l'ablette, la brème bordelière et le gardon).

Le premier plan factoriel $\mathrm{F} 1 \mathrm{~F} 2$ résume donc $49,9 \%$ de la variance du tableau faunistique. Un léger gradient d'abondance s'établit entre les stations, la station 1 étant la plus corrélée positivement à $F 1$, suivie du chenal principal (station 6). La station 4 est la dernière, mais elle héberge les plus fortes abondances en perche-soleil. La représentation des relevés dans ce plan (fig. 2C, 2D) exprime essentiellement un effet spatial. Trois groupes de stations se distinguent. Le premier groupe, dans la partie basse du plan formé par F1F2, rassemble les stations 5 et 6 qui sont les deux stations lotiques. A l'opposé, dans la partie haute du plan, les stations 2,3 et 4 , lentiques, constituent le deuxième groupe. Enfin, la station 1 est isolée et en position intermédiaire par rapport aux deux groupes précédemment définis. Cette station est lentique, mais largement ouverte sur le chenal comparativement aux autres stations lentiques.

Selon les stations, les relevés sont assez dispersés autour des centres de classe qui les représentent, ce qui indique qu'il existe des écarts entre les dates de pêche, pour une même station. Cette dispersion des relevés est particulièrement remarquable autour du centre figurant la station 1, ce qui tend à montrer que le peuplement piscicole y serait particulièrement variable au cours de la saison. 

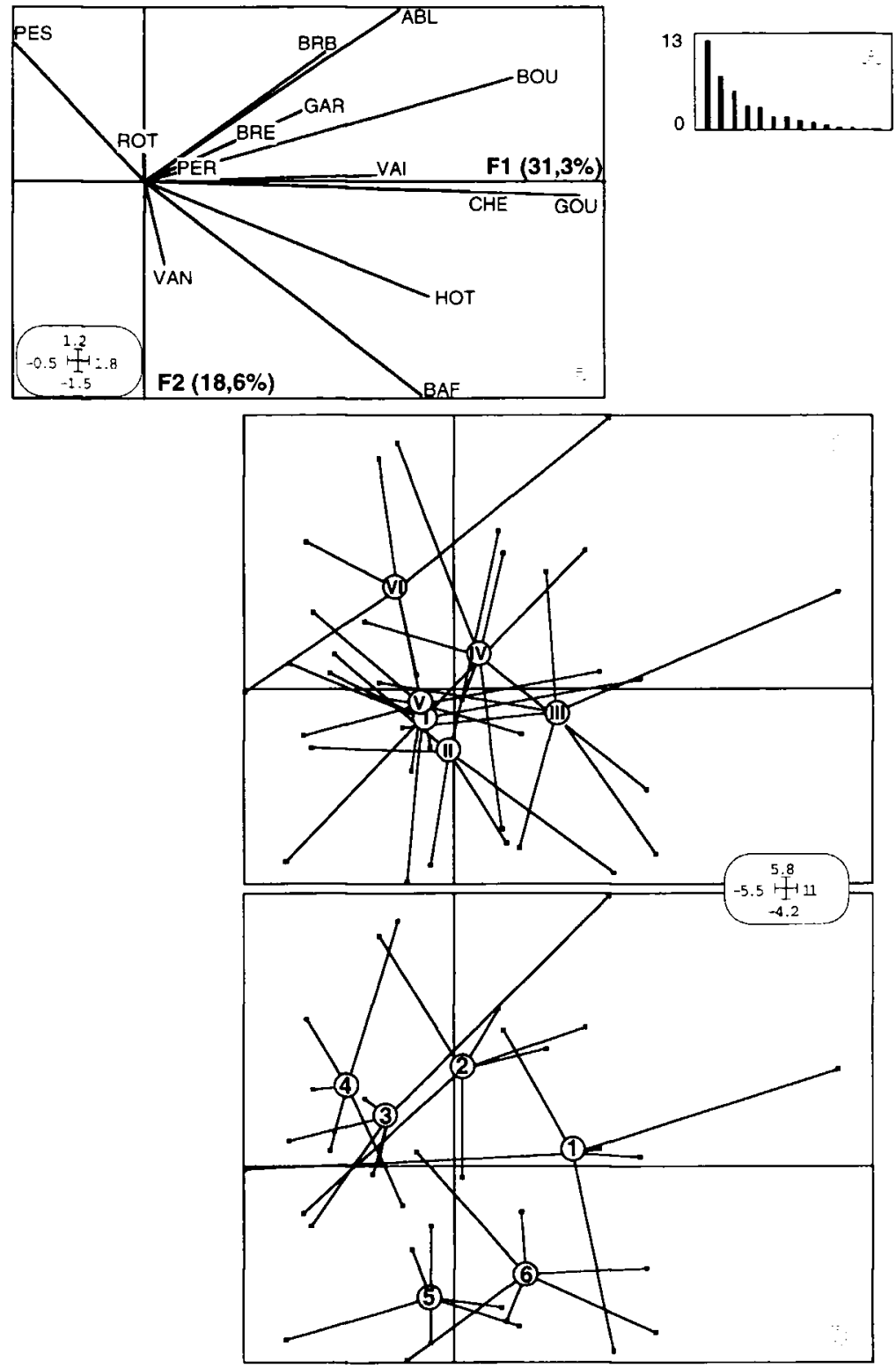

Figure 2 : Analyse en Composantes Principales centrée du tableau des abondances des espèces dans les relevés (14 espèces $\times 36$ relevés). A : graphe des valeurs propres ; $B$ : carte factorielle des espèces dans le plan F1F2 ; $C$ : carte factorielle dans le plan F1F2 des relevés et des centres de classe des relevés regroupés par date ; $D$ : carte factorielle dans le plan F1F2 des relevés et des centres de classe des relevés regroupés par station.

Figure 2 : Centred Principal Components Analysis of the table of species abundances by samples (14 species $\times 36$ samples station-date). A : Eigen values graph ; B : factorial map of species in F1F2; $C$ : factorial map of samples grouped by date in F1F2 ; $D$ : factorial map of samples grouped by site in F1F2.

La carte factorielle des relevés selon les dates (fig. 2C), montrant le regroupement des centres de classe vers l'origine des axes, ne met pas en évidence d'effet temporel prépondérant dans le plan F1F2. Seule la dernière campagne (date VI) s'écarte légèrement du groupe, vers la partie gauche du plan, indiquant la corrélation avec l'abondance tardive de la perche-soleil. La dispersion importante des relevés autour des centres de classe “ date » confirme les différences exprimées entre les stations. 
Le troisième axe F3 (fig. 3A), pris en compte dans l'analyse, extrait $13,5 \%$ de l'inertie totale du tableau. Deux espèces contribuent principalement à cet axe, le gardon et la vandoise. Ces espèces sont essentiellement présentes lors des deux premières campagnes.
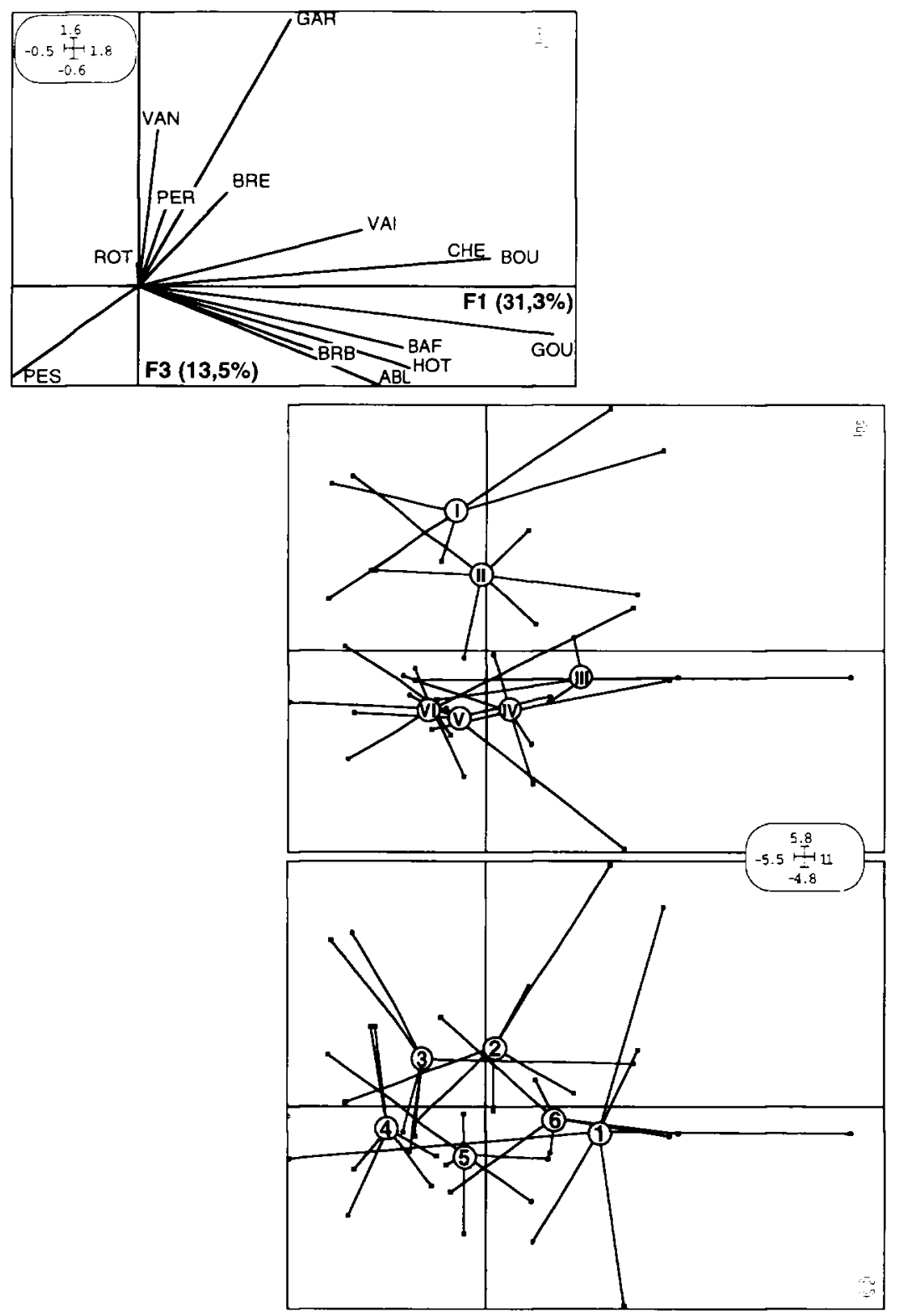

Figure 3 : Analyse en Composantes Principales centrée du tableau des abondances des espèces dans les relevés ( 14 espèces $\times 36$ relevés). $A$ : carte factorielle des espèces dans le plan F1F3 ; B : carte factorielle dans le plan F1F3 des relevés et des centres de classe des relevés regroupés par date; $C$ : carte factorielle dans le plan F1F3 des relevés et des centres de classe des relevés regroupés par station.

Figure 3 : Centred Principal Components Analysis of the table of species abundances by samples (14 species $\times 36$ samples station-date). A : factorial map of species in F1F3 ; B : factorial map of samples grouped by date in $\mathrm{F}^{\mathrm{F} 3} ; \mathrm{C}$ : factorial map of samples grouped by site in F1F3. 
La représentation des relevés par station (fig. $3 \mathrm{C}$ ) dans le plan $\mathrm{F} 1 \mathrm{~F} 3$ ne rend compte que du gradient d'abondance lié à l'axe $\mathrm{F} 1$ et évoqué précédemment. En revanche, la représentation par date des relevés dans ce plan (fig. 3B) confirme l'effet temporel en distinguant une opposition entre les deux premières dates et les suivantes, liée à l'axe F3. Néanmoins, les dates se succèdent chronologiquement dans ce plan. Cette succession exprime conjointement l'apparition des espèces selon leur période de reproduction, et leurs variations d'abondances qui ont tendance à décroître excepté pour la perche-soleil. L'axe F3 témoigne, en conséquence, de l'effet saisonnier auquel les peuplements des poissons de l'année sont soumis.

Ainsi, l'analyse des abondances des poissons de l'année permet de mettre en évidence les effets spatial et temporel maîtrisés dans le plan d'échantillonnage.

\section{Profils écologiques des espèces}

L'analyse fréquentielle des espèces permet de préciser la répartition de chaque espèce dans les stations et d'affiner particulièrement le gradient spatial dégagé par l'analyse multivariée. Les profils écologiques calculés peuvent être schématisés et exprimer la sur-représentation ou, à l'inverse, la sous-représentation de chacune des 14 espèces dans chaque station (fig. 4).

Les profils de trois espèces, le rotengle, la perche et la brème, n'ont pas été testés, car leur fréquence d'occurrence s'est avérée faible (inférieure à $5 \%$ ). Deux profils spécifiques se sont révélés non significatifs par le test du Khi2, ce qui indique que les deux espèces, la vandoise et le gardon, ne semblent pas manifester, dans le contexte étudié, de préférence vis-à-vis d'une ou plusieurs stations.
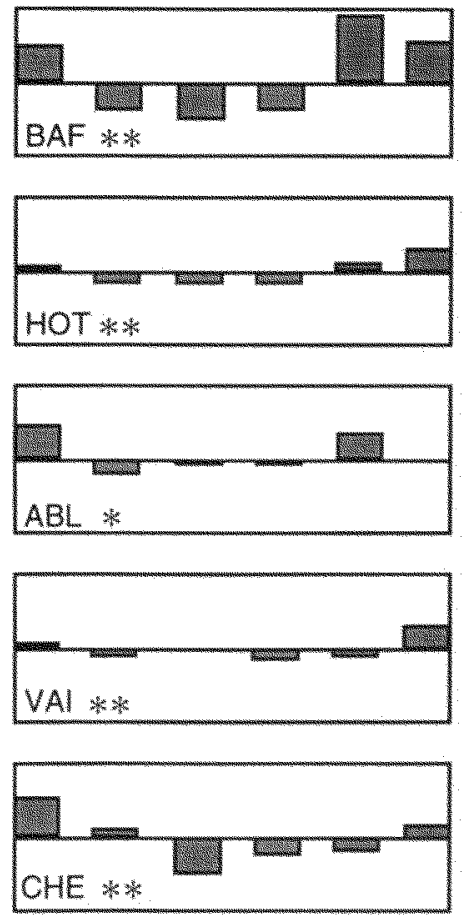
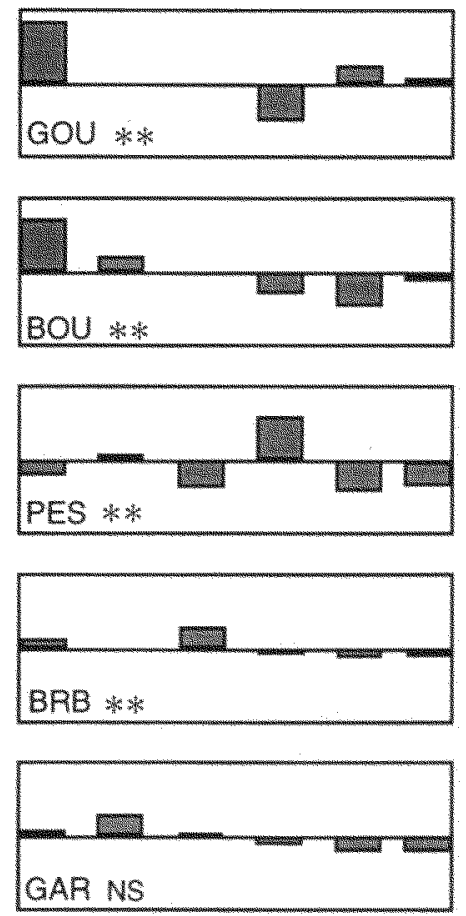
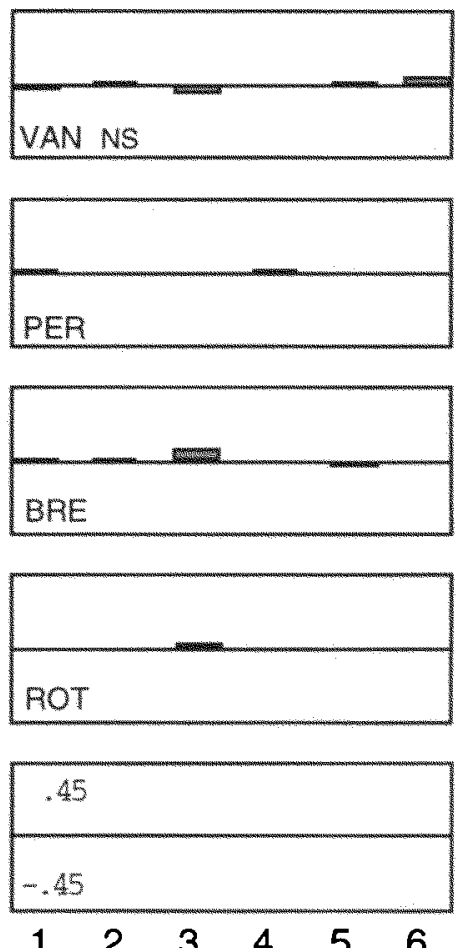

Figure 4 : Profils écologiques des espèces vis-à-vis des stations d'étude. NS : profil non significatif ; * : profil significatif $(P<0,05)$; ${ }^{* *}$ : profil très significatif $(P<0,01)$ (Test du $\left.\aleph^{2}\right)$.

Figure 4 : Ecological profiles of species versus study sites. NS : no significative profile ; ${ }^{\star}$ : significative profile $(P<0.05) ;{ }^{\star \star}$ : very significative profile $(P<0.01)$ (by $x^{2}$ Test). 
En revanche, neuf espèces ont des profils significatifs $(P<0,05)$ ou très significatifs $(P<0,01)$ qui peuvent être regroupés en plusieurs types. II est intéressant de les analyser en les confrontant à leurs guildes de reproduction (Tab. II).

Le premier type rassemble cinq espèces qui ont une tendance plus ou moins prononcée à être sur-représentées dans les stations lotiques (station 5 et chenal) et la station 1, et au contraire sous-représentées dans les stations lentiques (stations 2, 3 et 4). Toutes ces espèces sont rhéophiles et appartiennent au groupe des lithophiles, excepté l'ablette, plus généraliste, qui est eurytope et appartient au groupe des phytolithophiles. Le barbeau et le hotu ont les profils les plus caractéristiques et sont systématiquement sous-représentés dans les trois stations lentiques. Dans une moindre mesure, l'ablette présente un profil similaire mais ne manifeste pas de préférence particulière vis-à-vis du chenal, contrairement aux deux autres espèces. Le profil du vairon s'apparente également à ce groupe, mais a une affinité négative avec la station 5 . Enfin, le chevaine se distingue quelque peu au sein de ce groupe par ses affinités plus nuancées par rapport aux deux types de stations : il est légèrement sur-représenté dans la station 2, lentique, et sous-représenté dans la station 5 , lotique.

Le deuxième type de profil, caractérisé notamment par une valeur positive à la station 1 et négative à la station 4, rapproche deux espèces, le goujon et la bouvière. Ces deux espèces appartiennent pourtant à des groupes de reproduction différents : le goujon est l'unique représentant du groupe des psammophiles, et la bouvière, de celui des ostracophiles. Mais leurs profils diffèrent pour la station 5, lotique, dans laquelle le goujon, rhéophile, est sur-représenté contrairement à la bouvière, limnophile.

Enfin, deux espèces, la perche-soleil et la brème bordelière, ont des profils écologiques singuliers, qui ne prennent une valeur positive que dans une seule station. La perche-soleil est la seule de toutes les espèces capturées, nettement sur-représentée dans la station 4 et sousreprésentée partout ailleurs. Peu exigeante pour son support de ponte, elle est qualifiée de polyphile et appartient au groupe des nidificateurs (nest-guarders). Le profil écologique de la brème bordelière est également caractéristique. D'allure plate, il n'indique une sur-représentation de cette espèce, appartenant au groupe des phytophiles stricts, que dans la station 3.

L'examen des profils écologiques des espèces de poissons de l'année dans les différentes stations permet donc de retrouver la discrimination entre sites lotiques et lentiques. Mais, en les confrontant à l'appartenance des espèces aux différents groupes reproducteurs, il a été en outre possible d'affiner cette typologie sommaire.

\section{DISCUSSION ET CONCLUSION}

Les peuplements de poissons de l'année, dans l'ensemble des milieux, sont soumis à une évolution saisonnière qui correspond au rythme d'apparition des espèces selon leur période de reproduction. Globalement, cette évolution est conforme aux successions d'espèces mises en évidence par l'étude des rythmes de dérive des jeunes poissons dans le Rhône (OLIVIER, 1992) ou d'autres exemples de suivis saisonniers (SCHEFFEL et SCHIRMER, 1991). Simultanément, les abondances de jeunes de l'année ont tendance à décroître au fil de la saison. Cette diminution est vraisemblablement consécutive à la mortalité naturelle particulièrement sévère chez les jeunes stades de poissons. Mais, en grandissant, les poissons développent également leur capacité de mobilité, ce qui leur permet, d'une part, d'émigrer vers l'ensemble du secteur et, d'autre part, de fuir et d'éviter d'être capturés. L'effet saisonnier mis en évidence est toutefois secondaire par rapport à l'effet spatial analysé.

Dans l'ensemble des milieux étudiés, les peuplements des jeunes poissons de l'année se répartissent selon leur degré de rhéophilie et répondent à un gradient spatial qui évolue des milieux lotiques aux milieux lentiques. Un milieu intermédiaire, que l'on peut qualifier de semilotique, présente toutes les conditions des milieux lentiques en étant fortement ouvert sur le chenal. Ce gradient spatial se manifeste également vis-à-vis des groupes de reproduction constitués par les espèces piscicoles. 
Les milieux lotiques (chenal principal et chenal secondaire) sont globalement favorables aux espèces rhéophiles ou eurytopes à reproduction lithophile ou phytolithophile. Le milieu semilotique se révèle propice simultanément pour les groupes psammophile et ostracophile, qui sont représentés respectivement par une espèce rhéophile, le goujon, et une espèce limnophile, la bouvière. Cette affinité commune, a priori singulière, est liée à leur milieu de reproduction constitué de bancs de sable. Ces derniers constituent le substrat de ponte de la première espèce et l'habitat favori du mollusque bivalve dans lequel pond la seconde. Enfin, les milieux lentiques sont strictement caractérisés par les espèces limnophiles ou eurytopes. Des distinctions sont toutefois possibles entre ces derniers sites, où domine tantôt le groupe des phytolithophiles ou celui des phytophiles, voire celui des nidificateurs (nest-guarders). Cette complémentarité entre les milieux d'une plaine alluviale vis-à-vis de la reproduction des espèces piscicoles, a été mise en évidence précédemment dans le bassin du Danube (WITKOWSKI, 1984 ; HOLCIK, 1990 ; SCHIEMER et al., 1991) et dans le bassin du Rhône (COPP, 1989b).

Ce gradient spatial, le long duquel les espèces piscicoles se succèdent selon leur degré de rhéophilie et leur stratégie de reproduction, est assimilable à une zonation latérale du fleuve, par analogie à la zonation piscicole longitudinale. Cette zonation latérale est globalement conforme à celle établie sur le Rhône, en contexte peu anthropisé (COPP et PENAZ, 1988), laquelle répond à la typologie géomorphologique de sa plaine alluviale (AMOROS et al., 1982). En s'écartant du chenal principal, une variété de milieux annexes issus de ces déplacements se distingue. Ils correspondent à des stades d'évolution particuliers : les milieux les plus anciens sont les plus éloignés du chenal mais aussi les moins soumis aux échanges hydrauliques qui régissent le fonctionnement de l'hydrosystème. Ce rapprochement de situation entre les secteurs du Rhône et de la Seine témoigne, par conséquent, de l'état relativement préservé du secteur de la Seine étudié.

Dans ce secteur néanmoins, des différences entre les milieux d'origine naturelle ou anthropique sont décelables. Dans le cas des milieux lotiques, si le chenal secondaire diffère du chenal principal par un peuplement de poissons de l'année moins riche et moins diversifié, ceci est vraisemblablement lié principalement à leur différence de taille (PATTON et HUBERT, 1993). En revanche, pour les milieux lentiques, les effets de l'anthropisation sont plus marqués sur les peuplements. Le bras mort d'origine anthropique (station 4), isolé à l'amont par une digue, se distingue par de faibles abondances en poissons de l'année et ne paraît favorable qu'à une seule espèce, la perche-soleil, qui appartient au groupe des nidificateurs. Ce groupe sélectionne pour s'y reproduire des milieux peu soumis à des variations de niveau, comme ce bras mort dont les caractéristiques morphométriques lui confèrent une relative stabilité. Néanmoins, l'ensemble du secteur répond à ce critère de par sa situation à l'amont immédiat d'un barrage. Dans le Rhône amont, le groupe des nidificateurs caractérise les milieux annexes les plus anciens (COPP et PENAZ, 1988), où les apports allochtones sont réduits et les processus autotrophes, prédominants, ce qui correspond au bras mort considéré. Relativement isolé, celui-ci est en effet peu soumis aux apports externes provenant du chenal, sa communication étant réduite au regard de sa taille. Par ailleurs, la digue élevée dans sa partie amont empêche, même en période de crue, le passage de l'eau. Par rapport au schéma d'évolution des milieux annexes lentiques, les actions d'aménagement ont vraisemblablement entraîné son isolement brutal et son évolution anticipée vers un stade de sénescence précoce.

Dans des secteurs plus anthropisés tels le Rhône aval (POIZAT, 1993), la diversité des milieux se réduit grossièrement à deux types de milieux contrastés, lotiques et lentiques, et la structure du peuplement piscicole s'en trouve modifiée. Seules les espèces capables d'utiliser des annexes artificielles, telles des gravières en eau, subsistent en l'absence d'annexes naturelles diversifiées. Les travaux d'aménagement hydraulique peuvent aussi, en modifiant les faciès d'écoulement du chenal, réduire ou supprimer la disponibilité en milieux lotiques, ce qui, à terme, met en danger la composante rhéophile et lithophile du peuplement piscicole (HOLCIK, 1990).

Par conséquent, la diversité des types de milieu de la plaine alluviale est nécessaire à la reproduction de toutes les espèces de poissons susceptibles d'y vivre. A ce titre, la préservation des milieux de type intermédiaire, propices à la fois aux espèces rhéophiles 
et lénitophiles, semble particulièrement importante, d'autant qu'ils représentent numériquement l'essentiel des milieux annexes. Dans les secteurs où ils ont disparu et où seule leur restauration est envisageable, des études préalables et complémentaires sont nécessaires pour identifier précisément l'influence de leurs caractéristiques d'habitat sur la reproduction des espèces de poissons. En revanche, dans des secteurs comparables au secteur étudié, il est essentiel de veiller au maintien même temporaire de la connexion entre le chenal et ces milieux annexes, c'està-dire à leur mise en eau durant toute la saison de reproduction et d'élevage des espèces de poissons. Ces milieux sont primordiaux, afin de préserver la biodiversité piscicole de l'ensemble de l'hydrosystème.

\section{REMERCIEMENTS}

Les auteurs remercient chaleureusement Alain RUMEAU, garde-chef du Conseil Supérieur de la Pêche, pour son efficacité incomparable sur le terrain, ainsi que Patrice NOURY. IIs remercient également Jérôme BELLIARD pour ses conseils et relectures avisés. Ce travail a été réalisé dans le cadre du programme CNRS PIREN-Seine.

\section{BIBLIOGRAPHIE}

AMOROS C., RICHARDOT-COULET M., PAUTOU G., 1982. Les ensembles fonctionnels : des entités écologiques qui traduisent l'évolution de l'hydrosystème en intégrant la géomorphologie et l'anthropisation. Revue de Géographie de Lyon, 57, 49-62.

ANTIPA G., 1911. Die Biologie des Inundationsgebietes der unteren Donau und des Donaudeltas. Verhandlungen des VIII Internationalen Zoologen Kongresses zu Graz, 15-20, 163-208.

BALINSKY B.I., 1948. On the development of specific characters in cyprinid fishes. Proc. Zool. Soc. Lond., 118, 335-344.

BALON E.K., 1975a. Reproductive guilds of fishes : a proposal and definition. J. Fish. Res. Board Can., 32, 821-864.

BALON E.K., 1975b. Terminology of intervals in fish development. J. Fish. Res. Board Can., 32, $1663-1670$.

BOUVET Y., PATTEE E., MEGGOUGH F., 1985. The contribution of backwaters to the ecology of fish populations in large rivers. Preliminary results on fish migrations within a side arm and from the side arm to the main channel of the Rhône. Verh. Internat. Verein. Limnol., 22, 2576-2580.

CARREL G., 1986. Caractérisation physico-chimique du Haut-Rhône français et de ses annexes ; incidences sur la croissance des populations d'alevins. Thèse de doctorat, Univ. Claude Bernard-Lyon I, 144 p. + annexes.

CHESSEL D., DOLÉDEC S., 1991. ADE software : multivariate analysis and graphical display for environmental data. Univ. Claude Bernard-Lyon I, $131 \mathrm{p}$.

COPP G.H., 1989a. Electrofishing for fish lavae and 0+ juveniles : equipment modifications for increased efficiency with short fishes. Aqu. Fish. Mgmt., 20, 453-462.

COPP G.H., 1989b. The habitat diversity and fish reproductive function of floodplain ecosystems. Environmental Biology of Fishes, 26, 1-27.

COPP G.H., PENAZ M., 1988. Ecology of fish spawning and nursery zones in the flood plain, using a new sampling approach. Hydrobiologia, 169, 209-224.

COPP G.H., OLIVIER J.M., PENAZ M., ROUXA.L., 1991. Juvenile fishes as functional describers of fluvial ecosystem dynamics : applications on the River Rhône, France. Regulated Rivers, 6, 135-145. 
CYR H., DOWNING J.A., LALONDE S., BAINES S.B., PACE M.L., 1992. Sampling larval fish populations : choice of sample number and size. Trans. Am. Fish. Soc., 121, 356-368.

DAGET P., GODRON M., 1982. Analyse de l'écologie des espèces dans les communautés. Masson, Paris, $163 \mathrm{p}$.

DIREN CHAMPAGNE-ARDENNE, 1995. Réseau National de Bassin, Bassin Seine-Normandie. Synthèse des résultats de l'annuaire 1994 et de l'évolution de la qualité générale de 1987 à 1994. Rapport DIREN, $14 \mathrm{p}$.

DZANA J.G., 1992. La Seine entre Bar-sur-Seine et Montereau : géomorphologie du fond de vallée et dynamique du lit mineur. Rapport PIREN-Seine, II/92/02, $30 \mathrm{p}$.

HOLCIK J., 1988. Influence of hydrological regime and water temperature on the activity and population density of fishes in the anabranches of the Danube. Prace Ust. Rybar. Hydrobiol., 6, 33-58.

HOLCIK J., 1990. Effects of hydraulic engineering on habitat and fish community in river anabranches of the middle Danube in VAN DENSEN W.L.T., STEINMETZ B., HUGHES R.H., Management of freshwater fisheries, 14-24, Pudoc, Wageningen.

KOBLITSKAIA A.P., 1981. Clef de détermination des alevins de poissons d'eau douce. Moscou, $208 \mathrm{p}$.

MOOIJ W.M., 1989. A key to the identification of larval bream, Abramis brama, white bream, Blicca bjoerkna, and roach, Rutilus rutilus. J. Fish Biol., 34, 111-118.

NELVA A., PERSAT H., CHESSEL D., 1979. Une nouvelle méthode d'étude des peuplements ichtyologiques dans les grands cours d'eau par échantillonnage ponctuel d'abondance. C.R. Acad. Sci., Paris, 679-791.

OLIVIER J.M., 1992. Rythmes de dérive des alevins en milieu fluvial (Vol. 1 et 2). Thèse de doctorat, Univ. Claude Bernard Lyon I, 129 p. + annexes.

PATTON T.M., HUBERT W.A., 1993. Reservoirs on a great plains stream affect downstream habitat and fish assemblages. J. Freshwater Ecol., 8, 279-286.

POIZAT G., 1993. Echelle d'observation et variabilité des abondances de juvéniles de poissons dans un secteur aval du Rhône. Thèse de doctorat, Univ. Cl. Bernard Lyon I, $217 \mathrm{p}$.

REIMER G., 1991. The ecological importance of floodplains for fish at the river March (Austria). Arch. Hydrobiol., 121, 355-363.

RICHARDOT-COULET M., AMOROS C., REYGROBELLET J.L., ROUX A.L., 1982. Diagnose des ensembles fonctionnels aquatiques définis sur le Haut-Rhône français. Application à une cartographie écologique d'un système fluvial. Eau du Québec, 15, 146-153.

SABO M.J., KELSO W.E., 1991. Relationship between morphometry of excavated floodplain ponds along the Mississipi river and their use as fish nurseries. Trans. Am. Fish. Soc., 120, 552-561.

SCHEFFEL H.J., SCHIRMER M., 1991. Larvae and juveniles of freshwater and euryhaline fishes in the tidal river Weser at Bremen, FRG. Verh. Internat. Verein. Limnol., 24, 2446-2450.

SCHEIDEGGER K.J., BAIN M.B., 1995. Larval fish distribution and microhabitat use in freeflowing and regulated rivers. Copeia, 125-135.

SCHIEMER F., SPINDLER T., WINTERSBERGER H., SCHNEIDER A., CHOVANEC A., 1991. Fish fry associations : important indicators for the ecological status of large rivers. Verh. Internat. Verein. Limnol., 24, 2497-2500.

SCHIEMER F., WAIDBACHER H., 1992. Strategies for conservation of a Danubian fish fauna in BOON P.J., CALOW P., PETTS G.E., Eds., River conservation and management, 363-382, John Wiley and Sons. 
SCOTT M.T., NIELSEN L.A., 1989. Young fish distribution in backwaters and main channel borders of the Kanawha river, West Virginia, J. Fish Biol., 35 (suppl. A), 21-27.

SHEAFFER W.A., NICKUM J.G., 1986. Backwater areas as nursery habitats for fishes in pool 13 of the upper Mississipi River. Hydrobiologia, 136, 131-140.

SPINDLER T., 1988. Bestimmung der mitteleuropaischen Cyprinidenlarven. Osterreichs Fisherei, 75-79.

WITKOWSKI A., 1984. Structure of communities and biomass of ichtyofauna in the Biebrza River, its old river beds and affluents. Pol. ecol. Stud., 10, 447-474. 